\title{
Robotic Colorectal Surgery: Is It Still on the Way to a Landmark?
}

\author{
Nam Kyu Kim \\ Department of Surgery, Yonsei University College of Medicine, Seoul, Korea
}

\section{See Article on Page 19-26}

Since a robotic system was first applied to colorectal cancer surgery at Severance Hospital in 2006, robotic surgery cases have increased rapidly, while 33 da Vinci robotic systems being installed at 27 hospitals and a total of 14,067 cases by the end of 2010 in Korea. Among the total cases, 6,365 cases (45\%) were in the field of general surgery, such as thyroid, stomach and colorectal surgery. Compared with laparoscopic surgery, Surgery with robotic systems is known to have several advantages, including a more ergonomic position, a stable camera platform, a stereoscopic view, elimination of tremors, and improved dexterity. The author, an experienced laparoscopic colorectal surgeon, analyzed his personal initial experiences with robotic and laparoscopic colorectal surgery. He found more positive results in robotic surgery than in laparoscopic surgery even though the parameters did not show any statistically significance differences. For example, a lower conversion rate, less blood loss, more harvested lymph nodes and better short-term clinical outcomes were noted in robotic series However, no cost-benefit-analysis data were presented in this article. These data are really in the same form as those in studies conducted during the initial period of laparoscopic surgery. The process of validating the feasibility and safety of robotic colorectal surgery seems to be following the same road map as that for laparoscopic surgery.

Many papers have presented comparative studies between robotic colorectal surgery and open colorectal surgery. Large clinical prospective randomized clinical trials have also been

Correspondence to: Nam Kyu Kim, M.D.

Department of Surgery, Yonsei University College of Medicine, 50 Yonsei-ro, Seodaemun-gu, Seoul 120-752, Korea

Tel: +82-2-2228-2117, Fax: +82-2-313-8289

E-mail:namkyuk@yuhs.ac

(C) 2012 The Korean Society of Coloproctology

This is an open-access article distributed under the terms of the Creative Commons Attribution NonCommercial License (http://creativecommons.org/licenses/by-nc/3.0) which permits unrestricted noncommercial use, distribution, and reproduction in any medium, provided the original work is properly cited. conducted. Most studies, including this study, have reported that robotic colorectal surgery is safe and feasible based on short-term outcomes [1-4]. Baek et al. [5], based on a threeyear follow-up, reported that robotic rectal surgery could be carried out safely in terms of recurrence and survival rates. However, all such studies are limited by small sample size and by the fact that they were conducted at a single institution.

The short-term and the long-term outcomes of robotic colorectal surgery will be verified based on these clinical studies and on any advantages of the new robotic system compared with the conventional laparoscopic system (fewer conversions, fewer complications, etc.), but the high cost for robotic systems and instruments still remains a problem for wide application. Thus, some additional studies that are quite different from laparoscopic clinical studies and that will study the merits of robotic surgery are needed. We still hunger for strong evidence regarding the advantages to patients and surgeons that will outweigh the high cost of robotic systems and equipment. Recently, in a prospective comparative study of a laparoscopic and a robotic TME, the urogential function was recovered earlier in the robotic TME than in the laparoscopic one [6]. Recently, the robotic versus laparoscopic resection for rectal cancer trial, a prospective randomized clinical trial comparing robotic-assisted and laparoscopic surgery for rectal cancer, was begun. In Korea, the COLRAR trial, a prospective, randomized, controlled trial of assess robotic-assisted surgery and laparoscopy assisted surgery in patients with mild- or low rectal cancer, was also recently begun. Even though a good landmark has not yet been found, we must be on the path to find such a landmark.

\section{REFERENCES}

1. Baik SH, Kwon HY, Kim JS, Hur H, Sohn SK, Cho CH, et al. Robotic versus laparoscopic low anterior resection of rectal cancer: short-term outcome of a prospective comparative study. Ann Surg Oncol 2009;16:1480-7.

2. Patriti A, Ceccarelli G, Bartoli A, Spaziani A, Biancafarina A, Casciola L. Short- and medium-term outcome of robot-assisted and traditional laparoscopic rectal resection. JSLS 2009;13:176-83. 
3. Bianchi PP, Ceriani C, Locatelli A, Spinoglio G, Zampino MG, Sonzogni A, et al. Robotic versus laparoscopic total mesorectal excision for rectal cancer: a comparative analysis of oncological safety and short-term outcomes. Surg Endosc 2010;24:2888-94.

4. Park JS, Choi GS, Lim KH, Jang YS, Jun SH. Robotic-assisted versus laparoscopic surgery for low rectal cancer: case-matched analysis of short-term outcomes. Ann Surg Oncol 2010;17:3195-202.

5. Baek JH, McKenzie S, Garcia-Aguilar J, Pigazzi A. Oncologic out- comes of robotic-assisted total mesorectal excision for the treatment of rectal cancer. Ann Surg 2010;251:882-6.

6. Kim JY, Kim NK, Lee KY, Huh H, Min BS, Kim JW. A comparative study of voiding and sexaul function after TME with antunomic nerve preservation for rectral cancer: lapa vs robotic surgery. Ann Surg Oncol DOI 10.1245/s1034-012-2262-1.2012 Feb 6 , in press. 